\title{
Structural Evolution in Zeolite Fluid Cracking Catalyst
}

Andrew Meng ${ }^{1}$, Ke-Bin Low ${ }^{2}$, Junmei $\mathrm{Wei}^{3}$, Nicholas Favate ${ }^{3}$, Thomas Gegan ${ }^{3}$, Ivan Petrovic ${ }^{3}$ and Eric $\operatorname{Stach}^{4}$

${ }^{1}$ University of Pennsylvania, United States, ${ }^{2}$ BASF Corporation, Iselin, New Jersey, United States, ${ }^{3}$ BASF Corporation, United States, ${ }^{4}$ University of Pennsylvania, Philadelphia, Pennsylvania, United States

The fluid catalytic cracking process with zeolite catalysts is used to produce a large fraction of the world's gasoline. Although the process has been utilized for many years, the field remains very active as mechanistic questions remain. In particular, the location of the catalytically active sites and the role of defects in the catalytic performance and material lifetime is unclear. Fluid cracking catalysts generally undergo an activation process involving steaming, which results in the formation of dealumination vein defects (Figure 1a-b). These defects in zeolites are interesting due to their influence on mesopore structure that could affect catalytic activity.[1] Furthermore, the behavior of structurally and chemically different regions of the zeolite catalyst during active catalytic conditions could provide insights into coke formation mechanisms.

Previous work has used EELS to characterize chemical differences in zeolites [2], and we combine this with in-situ TEM to investigate zeolite structural evolution. Combining in-situ electron microscopy with spatially resolved chemical analysis through electron energy loss spectroscopy (EELS) offers new insights into the structural evolution of defects in zeolites during chemical processes such as steaming, which is used to convert the material to the catalytically active phase, or hydrocarbon cracking, which occurs during active catalyst operation. In a model fluid cracking catalyst material, we find that steam activation-induced dealumination vein defects preferentially form in non-uniform regions of the zeolite with variation in composition and thickness. We perform operando hexane cracking and ex-situ decane cracking experiments to examine structural effects on coke formation. Using EELS to distinguish between the clay matrix, un-veined zeolite, and veined zeolite to better understand the different components of the fluid cracking catalyst, we investigate the differences in the formation of coke on these different regions. EELS maps of the coked and uncoked zeolite catalyst are shown in Figure 1(c-g). Furthermore, use of multiple linear least squares (MLLS) fitting of carbon EELS spectra of different regions of coked zeolite catalyst showed differences in the EELS spectra of carbon on the zeolite and off the zeolite (Figure 2). These results are important because it may be possible to link coke formation to regions of the zeolite catalyst with specific structural characteristics, which can provide insights on ways to reduce coke formation in fluid cracking catalysts. 

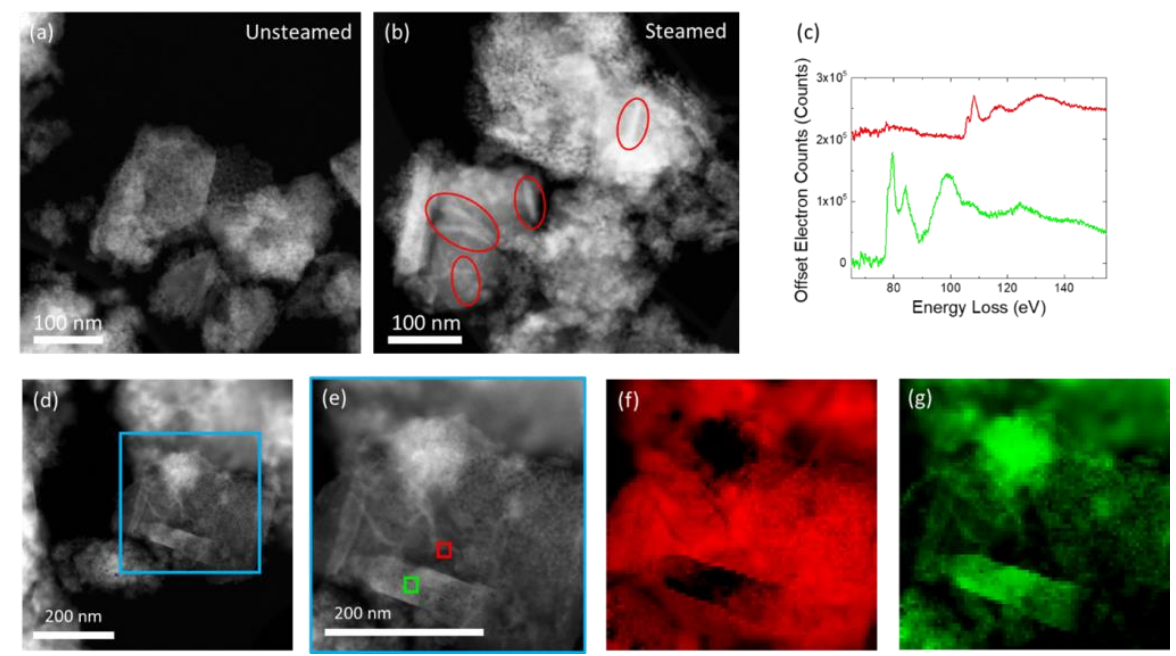

Figure 1. Dark field STEM images of a) unsteamed zeolite without dealumination veins, and b) steamed zeolite exhibiting dealumination veins c) EELS spectra of veined and unveined zeolite regions in d) dark field STEM image of steamed zeolite with marked regions of interest in e) magnified inset and f) MLLS fitting using reference spectra of areas marked in e) and shown in c)
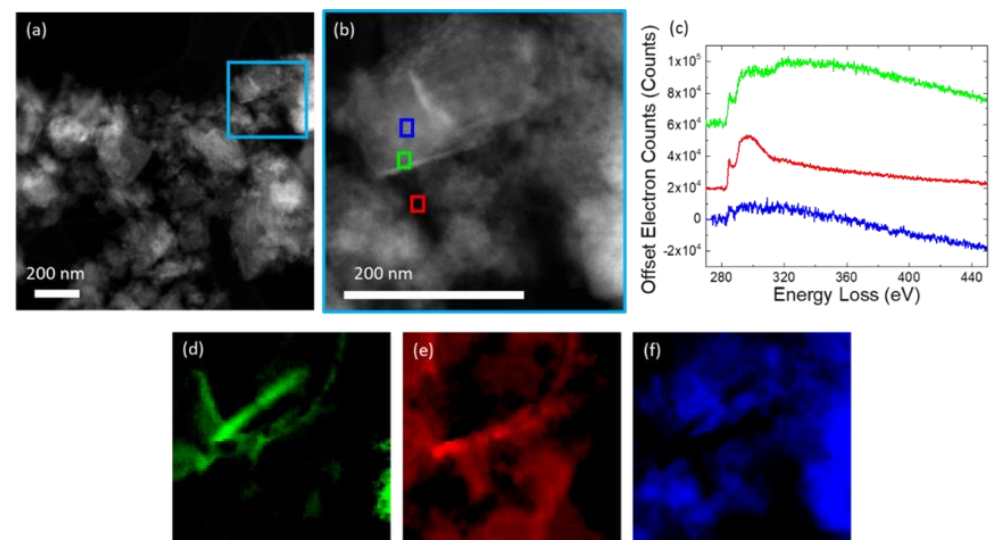

Figure 2. a) Dark field STEM image of coked zeolite after ex-situ decane cracking, b) magnified inset, c) electron energy loss spectra of C K-edge in marked rectangular regions of interest, C EELS map of d) coked-, e) off-zeolite and f)uncoked-zeolite according to MLLS fit

\section{References}

[1] P. Peng, X.-H. Gao, Z.-F. Yan, S. Mintova, Diffusion and catalyst efficiency in hierarchical zeolite catalysts, National Science Review, 7 (2020) 1726-1742.

[2] F. Paraguay D, M. Miki-Yoshida, F. Espinosa-Magaña, E. Terrés, J.M. Dominguez, Electron Energy Loss Spectroscopy Study of ZSM-5 Zeolite and MCM-41 Mesoporous Materials, Microscopy and Microanalysis, 7 (2001) 1072-1073. 